Dear author,

Please note that changes made in the online proofing system will be added to the article before publication but are not reflected in this PDF.

We also ask that this file not be used for submitting corrections. 


\section{Improving hydrogen production from the hydrolysis of ammonia borane by using multifunctional catalysts}

\section{R. Gil-San-Millan, A. Grau-Atienza, D.T. Johnson, S. Rico-Francés, E. Serrano, N. Linares *1, J. Garcia-Martinez ${ }^{* *, 1}$}

Molecular Nanotechnology Lab, Department of Inorganic Chemistry, University of Alicante, Carretera San Vicente s/ n, E-03690 Alicante, Spain

\section{A R T I C L E I N F O}

\section{Article history:}

Received 27 April 2018

Received in revised form

20 June 2018

Accepted 23 June 2018

Available online $\mathrm{xxx}$

\section{Keywords:}

Hydrogen storage

Ammonia borane hydrolysis

Hydrogen generation

Heterogeneous catalysis

Multifunctional catalyst

\begin{abstract}
A B S T R A C T
A novel multifunctional catalytic system has been developed for efficient hydrogen generation through the hydrolysis of ammonia borane. This system combines Pd NPs with acid sites and amines, which are both task-specific functionalities able to destabilize the $\mathrm{N} \rightarrow \mathrm{B}$ dative bond. The acidity of the support (zeolites of different structure and $\mathrm{SiO}_{2} / \mathrm{Al}_{2} \mathrm{O}_{3}$ ratio) used to disperse the Pd NPs causes an increase in the hydrogen production rate. However, the positive effect of incorporating $p$-phenylenediamine in the catalyst is much more pronounced, causing a two-fold increase in the activity of the catalyst. The combined effect of the different functionalities yields excellent performance in the hydrolysis of ammonia borane, greatly enhancing the activity of the metal-based catalyst and reducing the activation energy of the catalyzed reaction.
\end{abstract}

๑) 2018 Published by Elsevier Ltd on behalf of Hydrogen Energy Publications LLC.

\section{Introduction}

Ammonia borane $(A B)$ is considered one of the most promising candidates for on-board hydrogen storage applications thanks to its high hydrogen content (19.6 wt\%), superior stability under ambient conditions, and non-toxicity [1,2]. One route for the dehydrogenation of $A B$ is its hydrolysis. In this case, the release of molecular hydrogen can be conveniently controlled by using acid or transition metal-based catalysts $[3,4]$. Equation (1) shows the reaction for the hydrolysis of $A B$ in excess of water [5]. There is broad agreement that the metal-catalyzed hydrolysis proceeds via complexation of the $\mathrm{N} \rightarrow \mathrm{B}$ dative bond with the metal surface, causing its destabilization and subsequent cleavage, which is the rate limiting step of this reaction. From there, the hydrolysis of $\mathrm{BH}_{3}$ proceeds rapidly to produce 3 moles of $\mathrm{H}_{2}$ per mole of $\mathrm{AB}[6,7]$.

$$
\mathrm{NH}_{3} \mathrm{BH}_{3}+4 \mathrm{H}_{2} \mathrm{O} \rightarrow \mathrm{B}(\mathrm{OH})_{4}^{-}+\mathrm{NH}_{4}^{+}+3 \mathrm{H}_{2} \uparrow
$$

Most of the research on the catalytic hydrolysis of $A B$ concerns the use of transition metal catalysts due to their

* Corresponding author.

** Corresponding author.

E-mail addresses: noemi.linares@ua.es (N. Linares), j.garcia@ua.es (J. Garcia-Martinez).

1 www.nanomol.es. 
higher activity [6-9]. Noble [10-14] and non-noble [7,15-18] metal catalysts have been evaluated being noble metals the ones showing superior performance and stability. However, the practical use of this system requires to reduce the use of the costly and scarce noble-based catalysts while maintaining their efficiency and recyclability [19]. In this regard, the alloying of two metals has been widely studied to address both the effectiveness and the costs of heterogeneous catalysts [20-22]. As a result, by controlling the size, morphology, and compositions of metal-based catalysts, the hydrogen production from $A B$ hydrolysis can be significantly enhanced [21].

In addition to metals, Lewis acid or basic sites can efficiently destabilize the $A B$ molecule leading to improved dehydrogenation rates [23-25]. For example, the functionalization of a metal-organic framework (MOF) like MIL-101(Cr) with amine and amide groups improved its catalytic activity for the dehydrogenation of $A B$ through thermolysis [26]. It has been suggested that the destabilization of $A B$ occurs via the formation of a hydrogen bond between the lone pair of the amine group and the hydrogen of $-\mathrm{NH}_{3}$ of the $\mathrm{AB}$. This hydrogen bonding perturbs the distribution of charges in the $A B$, which thus becomes more reactive. Recently, similar results have been obtained for the hydrolysis of $A B[27,28]$. Ndoped catalysts featured higher activities than bare catalysts prepared using the same synthetic method. However, a systematic study on the effect of the amines in a similar aminefunctionalized heterogeneous catalyst for the hydrolysis reaction of $A B$ has not been reported thus far. In a similar way, whilst the use of acid solids has proved to be effective in the catalyzed hydrolysis of $A B$ [29], the role of acidic supports in metal-based heterogeneous catalysts has not been fully explored [30] and only the sodium form of the zeolites has been explored so far [31-34].

Herein, we present the preparation of a multifunctional catalyst for the hydrolysis of $A B$ that contains various functionalities each of them able to destabilize the $\mathrm{N} \rightarrow \mathrm{B}$ dative bond and thus enhancing the hydrogen production rate. The combined use of noble metal nanoparticles with terminal amines and acid sites (through the use of zeolites as supports) has been evaluated. A systematic study of the effect of each functionality in this reaction has been carried out in order to prepare a multifunctional catalyst able to efficiently improve the catalytic activity of metal-based catalysts for this reaction. We propose that, as alloying of different metals can be used to improve the activity of the catalysts for $A B$ hydrolysis, the combination of metals with different functionalities can be used as an attractive alternative which is both cost efficient and scalable.

\section{Experimental section}

\section{Chemicals}

Palladium nitrate hydrate, ethylenediamine (ED), 4aminopyridine (APy), sodium tetrachloropalladate $\left(\mathrm{Na}_{2} \mathrm{PdCl}_{4}\right.$, 98\%), 4-(dimethylamino)pyridine (DMAP, $\mathrm{C}_{7} \mathrm{H}_{10} \mathrm{~N}_{2}, 99 \%$ ), tetraoctylammonium bromide (TOAB, $\left.\left(\mathrm{N}_{(}\left(\mathrm{C}_{8} \mathrm{H}_{17}\right)_{4}\right) \mathrm{Br}, 98 \%\right)$, tetraethylorthosilicate (TEOS, 98\%), ammonium fluoride $\left(\mathrm{NH}_{4} \mathrm{~F}\right.$, 98\%), p-phenylenediamine (PPD) and ammonia borane (AB,
90\%) were purchased from Aldrich; sodium borohydride $\left(\mathrm{NaBH}_{4},>96 \%\right)$ from Fluka. Zeolites were supplied by Zeolyst International (Table 1). All chemicals were used as received without further purification.

\section{Synthesis of the catalysts using silica supports}

As the hydrolysis of $A B$ strongly depends on the dispersion of metal nanoparticles [35], colloidal Pd nanoparticles (NPs) of narrow particle size distribution were prepared and used as the active metal sites. First, colloidal Pd NPs were obtained in toluene by the reduction of $\left[\mathrm{PdCl}_{4}\right]^{2-}$ with $\mathrm{NaBH}_{4}$ and subsequent stabilization using tetraoctylammonium bromide (TOAB) as capping agent. The Pd NPs were then transferred to an aqueous solution using 4-dimethylaminopiridine (DMAP) as the transfer agent. The detailed synthetic method can be found in the ESI and in references [36,37]. In order to evaluate the effect of different amines on the activity of the Pd NPs, they were functionalized on silica, used here as an inert (nonacidic) support. The silica-based catalysts were prepared by incorporation of the selected functionalities during the sol-gel synthesis of the silica material. Different amines such as, pyridine (py), p-phenylenediamine (PPD) and various trialkoxysilane-containing amines: a primary amine (3aminopropyl triethoxysilane, APTES), pyridine (2-(2pyridylethyl) trimethoxysilane, pyTMS) and ethylenediamine ([3-(2-Aminoethylamino)propyl] trimethoxysilane, A2PTMS), were loaded into the silica (see Scheme $1 \mathrm{~A}$ for their structures). The nominal palladium concentration in the catalysts was $0.22 \mathrm{wt} \%$ and the metal to amines molar ratio used was $\mathrm{Pd}$ :amine $=1: 2$. A schematic representation of the synthesis procedure to prepare the silica-based catalysts, Pd_Amine@$\mathrm{SiO}_{2}$, is shown in Scheme $1 \mathrm{~A}$.

In a typical synthesis, $1.0 \mathrm{~mL}(4.48 \mathrm{mmol})$ of TEOS, $0.50 \mathrm{~mL}$ of an aqueous Pd NPs suspension (concentration approx. $11 \mathrm{mM}$ ) and $0.1 \mathrm{~mL}$ of a $0.425 \mathrm{M} \mathrm{p}$-phenylenediamine aqueous solution were stirred in $1.25 \mathrm{~mL}$ of absolute ethanol for $10 \mathrm{~min}$. To induce the silica gel formation, $0.125 \mathrm{~mL}$ of $\mathrm{NH}_{4} \mathrm{~F} 0.5 \mathrm{M}$ ( $0.065 \mathrm{mmol} \mathrm{NH}_{4} \mathrm{~F}$ ) was added to the abovementioned solution. The mixture was reacted at room temperature during 15 min under vigorous stirring [38,39]. The formed wet gel was air dried in order to obtained the final silica xerogel.

\section{Synthesis of the catalysts using zeolites as acidic supports}

In this case, the Pd NPs were directly loaded on various zeolites by ion exchange and subsequent reduction either with $\mathrm{NaBH}_{4}$ or in situ during the $\mathrm{AB}$ hydrolysis reaction. Zeolites with different structures and $\mathrm{SiO}_{2} / \mathrm{Al}_{2} \mathrm{O}_{3}$ ratios were tested in order to assess the role of acidic and textural properties of the support in the catalytic performance of the different catalysts, see Table 1 . The commercial zeolites were previously calcined at $550{ }^{\circ} \mathrm{C}$ for $4 \mathrm{~h}\left(2{ }^{\circ} \mathrm{C} \mathrm{min}-1\right)$ under air flow to transform the initial ammonium to its corresponding proton-form. The introduction of the $\mathrm{Pd}(\mathrm{II})$ cations in the zeolites was carried out by ion exchange with $\mathrm{Pd}\left(\mathrm{NO}_{3}\right)_{2} \cdot 2 \mathrm{H}_{2} \mathrm{O}$. These materials were labeled as $\mathrm{Pd}(\mathrm{II}) /$ Zeolite, where Zeolite indicates the type of the zeolite used as a support. The Pd(II)-containing zeolites were directly added to the reaction media where the metal precursor was in situ reduced by the ammonia borane. For 
Table 1 - Zeolites used in this study.

\begin{tabular}{|c|c|c|c|c|}
\hline Zeolite name & Zeolite framework & Commercial name $^{a}$ & $\mathrm{SiO}_{2} / \mathrm{Al}_{2} \mathrm{O}_{3}$ molar ratio ${ }^{\mathrm{a}}$ & Cation form $^{a}$ \\
\hline \multirow[t]{5}{*}{ ZSM-5 } & MFI & CBV 2314 & 23 & Ammonium \\
\hline & & CBV 3024E & 30 & Ammonium \\
\hline & & CBV 5524G & 50 & Ammonium \\
\hline & & CBV 8014 & 80 & Ammonium \\
\hline & & CBV 28014 & 280 & Ammonium \\
\hline USY & FAU & CBV 720 & 30 & Hydrogen \\
\hline Beta & BEA & CP814E & 25 & Ammonium \\
\hline Mordenite & MOR & CBV 21A & 20 & Ammonium \\
\hline Ferrierite & FER & CP914C & 20 & Ammonium \\
\hline
\end{tabular}

A) Silica-based catalysts:

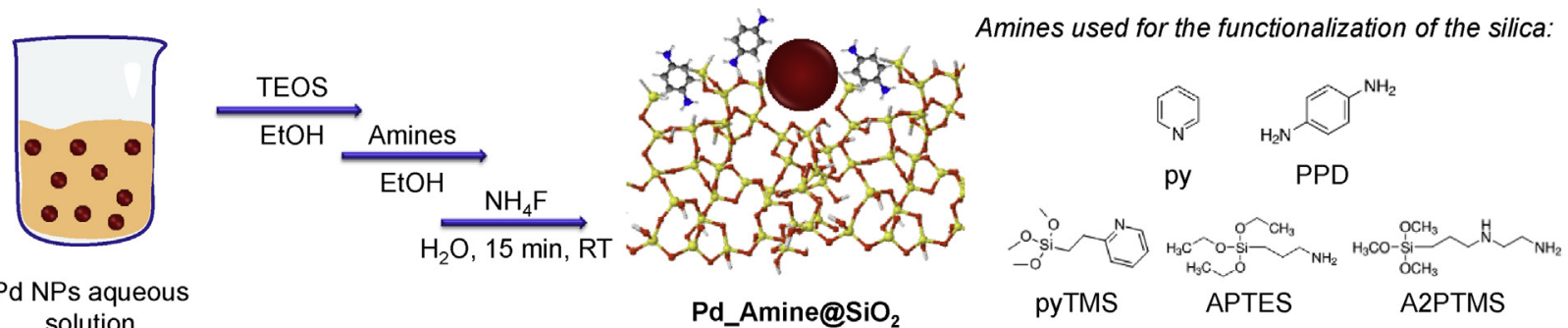

B) Zeolite-based catalysts:
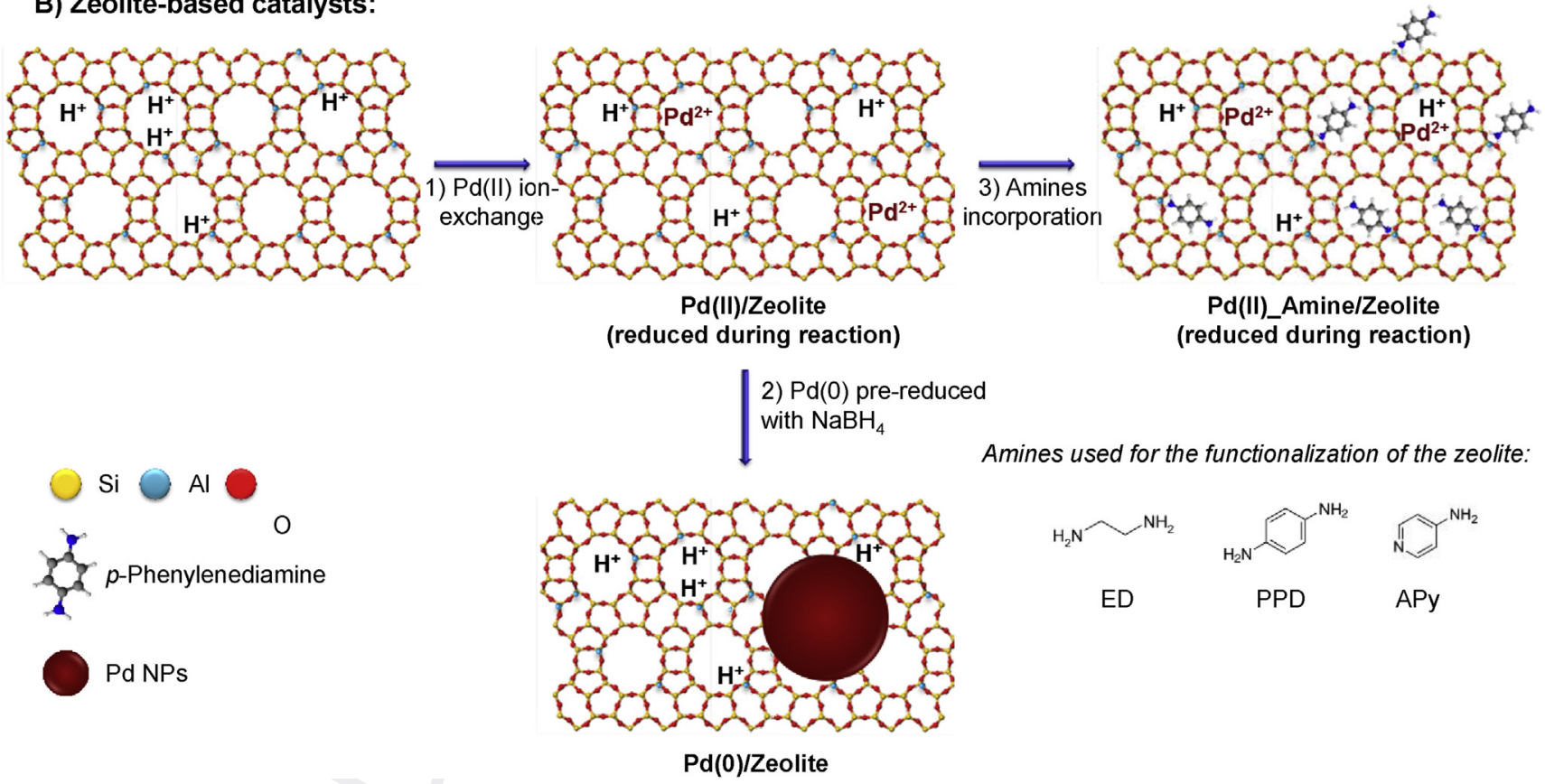

Scheme 1 - Schematic representation of the synthesis procedure followed to prepare the different Pd-catalysts evaluated: A) using silica as a support and B) using different zeolites as supports (beta zeolite and PPD have been chosen as examples). The legend for both schemes is shown in the left bottom corner. The structure of the amines used for the functionalization of the catalysts is shown next to the corresponding catalyst.

comparison purposes, the metallated zeolites were also converted into $\mathrm{Pd}(0)$-containing catalysts by previous reduction of the metal using an aqueous solution of $\mathrm{NaBH}_{4}$. Those materials where named as $\operatorname{Pd}(0) / Z$ Zeolite. Finally, several amine groups were incorporated to the $\mathrm{Pd}(\mathrm{II})$ catalysts in order to evaluate the combined effect of these compounds with metal and acid sites over the dehydrogenation of AB (Pd(II)_Amine/ Zeolite). The selected compounds were the diamines ethylenediamine (ED), 4-aminopyridine (APy), and p-phenylenediamine (PPD). A schematic representation of the different 
catalysts prepared is shown in Scheme 1B, where zeolite beta was used as an example.

In a typical synthesis, $21.9 \mathrm{mg}$ of $\mathrm{Pd}\left(\mathrm{NO}_{3}\right)_{2} \cdot 2 \mathrm{H}_{2} \mathrm{O}$ was first dissolved in $20 \mathrm{~mL}$ of water. Subsequently, the precursor solution was poured over $3.5 \mathrm{~g}$ of $\mathrm{H}$-zeolite, which corresponds to a $0.25 \mathrm{wt} \% \mathrm{Pd}$. The mixture was stirred for $1 \mathrm{~h}$, filtered, washed with distilled water and dried at $40{ }^{\circ} \mathrm{C}$ overnight to obtain the $\mathrm{Pd}(\mathrm{II}) /$ Zeolite materials. After the introduction of the Pd(II), the catalyst was stirred in an aqueous solution of the selected amine for $30 \mathrm{~min}$. The solid was subsequently filtered, washed with distilled water, and dried at $40{ }^{\circ} \mathrm{C}$ overnight, resulting in the $\mathrm{Pd}(\mathrm{II}) \_$Amine/Zeolite catalysts. In the case of the catalysts with pre-reduced Pd NPs, Pd(0)/Zeolite, the reduction was carried out using a $0.1 \mathrm{M}$ solution of $\mathrm{NaBH}_{4}$ with a molar ratio metal/ $\mathrm{NaBH}_{4}=0.7$.

\section{Catalytic hydrolysis of ammonia borane}

The catalytic activity of the materials for the hydrolysis of ammonia borane was determined by measuring the rate of hydrogen generation as follows. The catalyst (c.a. $75 \mathrm{mg}, 0.25 \mathrm{wt}$ $\% \mathrm{Pd}$ ) was transferred into a glass tube (13 mm diameter), which was sealed with a silicon septum, and placed in an oil bath thermostated at $30.0-55.0 \pm 0.1^{\circ} \mathrm{C}$. The tube exhaust was connected to a water-filled inverted measurement cylinder. In a typical experiment, ammonia borane $(10.8 \mathrm{mg}, 0.31 \mathrm{mmol})$ was dissolved in $855 \mu \mathrm{l}$ of water. To start the catalytic cycle, the freshly prepared ammonia borane solution was injected into the tube and the hydrogen generated was measured due to displacement of the liquid in the inverted measurement cylinder [40-42]. Time was controlled using a chronometer. The catalytic experiments were repeated at least three times in order to obtain reproducible data. The turnover frequency was determined in every case as: TOF $=\left(\mathrm{mol} \mathrm{H}_{2}\right.$ obtained $) /(\mathrm{mol}$ $\mathrm{Pd} \times \mathrm{min})$, calculated from the slope of the linear part of the $\mathrm{H}_{2}$ evolution us time plot, and using the bulk Pd content.

\section{Characterization}

The palladium content of each sample was determined by Inductively Coupled Plasma-Optical Emission Spectroscopy (ICP-OES) on a Perkin Elmer 7300 DV spectrometer. The samples were dissolved in aqua regia and the undissolved siliceous matter was filtered off $(0.45 \mu \mathrm{m})$ prior to the analysis.

The morphology of the catalysts and the size and distribution of Pd NPs was investigated by transmission electron microscopy (TEM) using a JEM-2010 microscope (JEOL, $200 \mathrm{kV}$, $0.14 \mathrm{~nm}$ of resolution). Samples were prepared by dipping a sonicated suspension of the sample in ethanol on a carboncoated copper prior to be studied by TEM.

The porous texture was characterized by $\mathrm{N}_{2}$ physisorption at $77 \mathrm{~K}$ in an AUTOSORB- 6 apparatus. The samples were previously degassed for $4 \mathrm{~h}$ at $523 \mathrm{~K}$ at $5 \times 10^{-5}$ bars. The surface area was calculated by using the BET method [43] and the total pore volume was estimated from the DFT method as described elsewhere [44].

$\mathrm{X}$-ray diffraction patterns were collected on a Seifert XRD 3003 TT (Bragg-Brentano geometry) powder diffractometer using a Cu K $\alpha$ radiation $(\lambda=1.54056 \AA)$ at a scanning velocity of $0.05^{\circ} \mathrm{min}^{-1}$ in the $2.5^{\circ}<2 \theta<50^{\circ}$ range.
The organic content of the amine-containing catalysts was measured by elemental combustion analysis on a Carlo Erba CHNS-O EA1108 analyzer. Complementary, thermogravimetric analyses (TGA) were carried out in a simultaneous TGDTA apparatus METTLER TOLEDO model TGA/SDTA851and/ $\mathrm{SF} / 1100$. Analyses were performed in a $\mathrm{N}_{2}: \mathrm{O}_{2}=4: 1$ atmosphere from room temperature to $1100{ }^{\circ} \mathrm{C}\left(10^{\circ} \mathrm{C} \mathrm{min}{ }^{-1}\right)$.

The incorporation of the amines in the catalysts was studied by IR spectra. The analyses were carried out on a Nicolet Nexus FT-IR Spectrometer. All the samples were prepared as $\mathrm{KBr}$ diluted pellets.

\section{Results and discussion}

\section{Silica support for the immobilization of Pd nanoparticles and} amines

The colloidal Pd NPs obtained using the procedure aforementioned present a narrow particle size distribution $(2.8 \pm 0.9 \mathrm{~nm})$, see Fig. S1. Further details regarding the characterization of the colloidal Pd NPs are given in the ESI (Figs. S1-S3). The small size and the low content of the Pd NPs immobilized on the heterogeneous catalysts make their characterization by TEM and XRD challenging (Figs. S4-S5). Regarding the textural properties of the silica catalysts, all the solids show type IV isotherms with BET surface areas and total pore volumes ranging from 210 to $250 \mathrm{~m}^{2} \mathrm{~g}^{-1}$ and $0.54-0.60 \mathrm{~cm}^{3} \mathrm{~g}^{-1}$, respectively, and broad pore size distributions (see Fig. S6). The Pd content in the solids was determined by ICP and EDX analyses, resulting in loading values very similar to the theoretical ones.

The immobilization of the Pd NPs on the silica support $\left(\mathrm{Pd} @ \mathrm{SiO}_{2}\right)$ has a significant effect on their catalytic performance. The heterogenized system was significantly more efficient in the generation of $\mathrm{H}_{2}$ (expressed as TOF) than $\mathrm{Pd}$ NPs in a colloidal suspension, 8.9 and $5.9 \mathrm{~min}^{-1}$, respectively (see Fig. S8). This improvement was attributed to the higher stability of the supported Pd NPs. The low stability of unsupported colloidal Pd NPs during reaction was evidenced by their agglomeration during their use (visible even to the naked eye). This led to the decrease of the $\mathrm{H}_{2}$ generation rate during the reaction, resulting in a nonlinear $\mathrm{H}_{2}$ production.

The role of the amines in the catalysis was evaluated by using terminal amines, such as py and PPD, and amines with terminal trialkoxysilane groups, such as APTES, pyTMS and

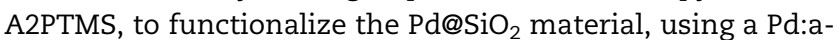
mine molar ratio $=1: 2$ (Fig. S7). Fig. 1 shows the TOF values of $\mathrm{H}_{2}$ generation obtained using these bifunctional catalysts (TOF values were determined from the slope of the linear region of the $\mathrm{H}_{2}$ evolution plot, Fig. S9). In general, the presence of the amines increased the rate of the generation of $\mathrm{H}_{2}$. Terminal primary amines, such as APTES and PPD, have a clear positive impact on the catalytic activity, accelerating quite notably the reaction, while pyridine and ethylenediamine have little impact on the hydrolysis of $A B$. The highest value was obtained for the Pd_PPD@SiO $\mathrm{S}_{2}$, whose activity is 3 times higher than the monofuncional Pd@SiO ${ }_{2}$ catalyst (see Fig. 1). It is worth pointing out that when only amines are added to the reaction mixture, no $\mathrm{H}_{2}$ evolution was observed. 


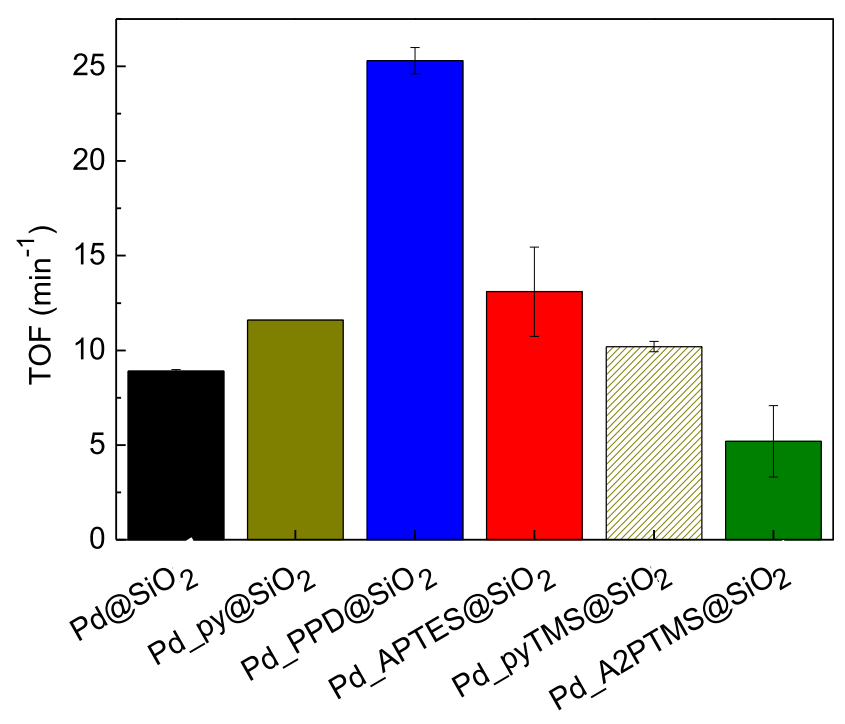

Fig. 1 - TOF values $\left(\min ^{-1}\right)$ for bifunctional Pd_AMINE@SiO ${ }_{2}$ catalysts prepared with the different amines named in the legend.

The $\mathrm{H}_{2}$ generation, then, depends on the metal catalyst, which is responsible for the breaking of the dative bond, the first step in the dehydrogenation of $\mathrm{AB}[5,6]$. As suggested by Gao et al. [20] for thermolysis, a hydrogen bond between the lone pair of the amine functionalized catalysts and the hydrogen of $-\mathrm{NH}_{3}$ on $A B$ can modify the distribution of charges, which leads to the acceleration of the $A B$ dehydrogenation. In this regard, it is well-known that aromatic amines, as PPD, are able to develop strong hydrogen-bonding interactions through their amine groups $[45,46]$. To the best of our knowledge, this feature has not been explored for the $A B$ hydrolysis reaction thus far.

\section{Zeolites as acidic supports for Pd nanoparticles}

To study the effect of supports containing acid sites, bifunctional acid/metal catalysts were firstly prepared using ZSM-5

a)

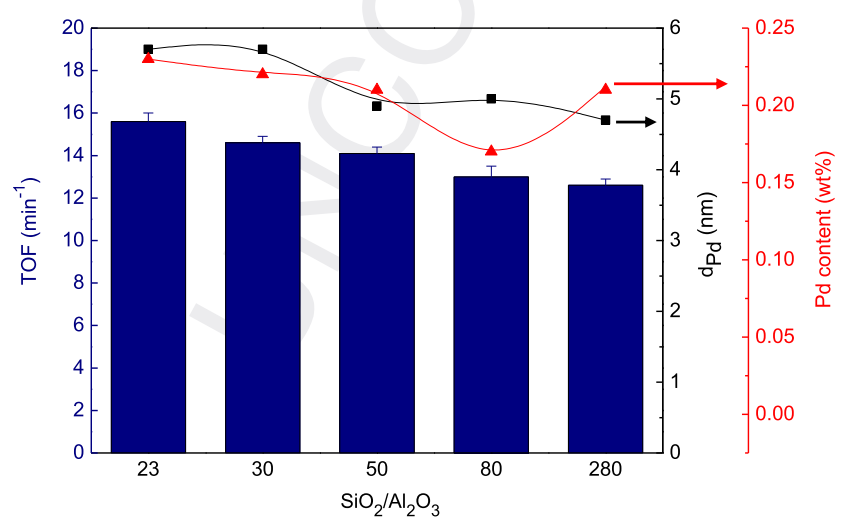

zeolites (MFI structure) with different $\mathrm{SiO}_{2} / \mathrm{Al}_{2} \mathrm{O}_{3}$ ratios, ranging from 23 to 280 ( $\mathrm{Pd}(\mathrm{II}) / \mathrm{ZSM}-5$ catalysts). Thus, the effect of using zeolites with increasing $\mathrm{Al}$ content, which is related to their total acidity $[47,48]$, as supports for the bifunctional catalysts was evaluated for ZSM-5 zeolite (Fig. 2 and S10). The Pd loading determined by ICP-OES was similar in all the catalysts, $0.17-0.23 \mathrm{wt} \%$, as well as the BET surface area, ca. 350-400 $\mathrm{m}^{2} \mathrm{~g}^{-1}$ in all cases (Fig. S11). The Pd(II)-zeolites were directly mixed with the $\mathrm{AB}$ solution, which was taken as the start time of the catalytic reaction. The Pd(II) was immediately in situ reduced during the catalytic tests and no induction period was observed in the hydrolysis of $A B$. Furthermore, the amount of $\mathrm{H}_{2}$ generated during the catalytic tests was not affected by the in situ reduction of the $\mathrm{Pd}(\mathrm{II}) \mathrm{as}$, in all the experiments, nearly 3 moles of $\mathrm{H}_{2}$ were produced per mole of AB. Fig. 2(a) shows TOF values for the tested zeolite-based catalysts under the same reaction conditions. As observed, the hydrogen production rate slightly increased with the $\mathrm{Al}$ content of the zeolite. Taking into account that all the zeolites have similar Pd content and particle size (see Fig. 2(a)), the differences in the hydrogen production rate should be attributed to the number of acid sites in the zeolites. In fact and as shown in Fig. 2(b), the TOF values follow a linear relationship with the relative $\mathrm{Al}$ content of the zeolites. A control experiment in which only bare zeolites.

Secondly, the effect of the zeolite structure in the hydrolysis of $A B$ was evaluated. In this case, $\mathrm{Pd}(\mathrm{II}) / \mathrm{Zeolite}$ catalysts made of zeolites with similar $\mathrm{SiO}_{2} / \mathrm{Al}_{2} \mathrm{O}_{3}$ ratios but different crystalline structure were prepared and tested (see Fig. S12 for $\mathrm{XRD}$ analyses). Table 2 shows the $\mathrm{SiO}_{2} / \mathrm{Al}_{2} \mathrm{O}_{3}$ ratio, Pd content, $\mathrm{BET}$ surface area, and catalytic results of the different catalysts studied. As can be observed, for similar Pd contents very different $\mathrm{H}_{2}$ production rates were obtained (Fig. S13), thus showing that the zeolite structure greatly influences the performance of the multifunctional catalyst. In this sense, the catalyst based on beta zeolite was the best performing one, leading to a three-fold increase in the rate of hydrogen production over zeolites ZSM-5, USY and mordenite. The higher

b)

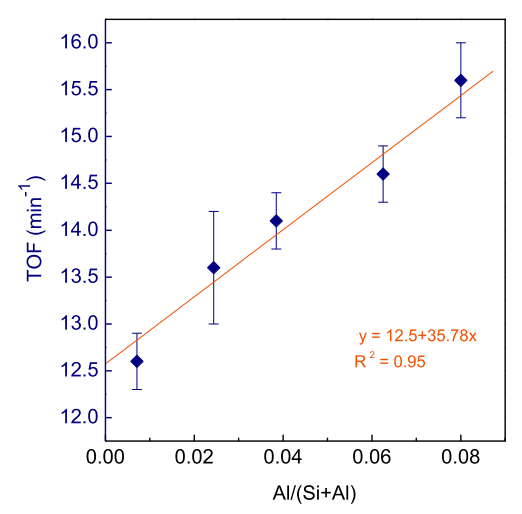
Web version of this article.) 
Table 2 - Properties and catalytic activity of $\mathrm{Pd}(\mathrm{II}) / Z$ /Zolite catalysts prepared by ion-exchange of $0.25 \mathrm{wt} \%$ of $\mathrm{Pd}(\mathrm{II})$ on different zeolites and reduced in situ during reaction.

\begin{tabular}{|c|c|c|c|c|c|}
\hline Zeolite & $\mathrm{SiO}_{2} / \mathrm{Al}_{2} \mathrm{O}_{3}$ & Pd loading ${ }^{a}(w t \%)$ & $\mathrm{S}_{\mathrm{BET}}^{\mathrm{b}}\left(\mathrm{m}[2] \cdot \mathrm{g}^{-1}\right)$ & $\mathrm{d}_{\mathrm{Pd}}{ }^{\mathrm{c}}(\mathrm{nm})$ & $\mathrm{TOF}^{\mathrm{d}}\left(\mathrm{min}^{-1}\right)$ \\
\hline ZSM-5 & 30 & 0.23 & 340 & $5.7 \pm 2.4$ & $14.6 \pm 0.3$ \\
\hline USY & 30 & 0.27 & 695 & $8.2 \pm 4.3$ & $18.3 \pm 0.1$ \\
\hline Beta & 25 & 0.27 & 500 & $2.2 \pm 0.9$ & $45.3 \pm 0.5$ \\
\hline Ferrierite & 20 & 0.26 & 455 & $3.7 \pm 1.5$ & $29.4 \pm 0.2$ \\
\hline Mordenite & 20 & 0.26 & 290 & $4.4 \pm 3.2$ & $13.1 \pm 0.6$ \\
\hline $\begin{array}{l}\text { a Pd conter } \\
\text { b The BET } \\
\text { c Particle s } \\
\text { d Reaction }\end{array}$ & $\begin{array}{l}\text { hed by ICP-O } \\
\text { a was estim } \\
\text { ation obtaine } \\
0.855 \mathrm{~mL} \text { o }\end{array}$ & $\begin{array}{l}\text { e multipoint } \mathrm{BET} \text { met } \\
\text { e analysis of at least } \\
\text { molar ratio } \mathrm{AB} / \mathrm{Pd}=\end{array}$ & $\begin{array}{l}\text { the adsorption dat } \\
\text { articles in TEM ima } \\
5^{\circ} \mathrm{C} \text {. }\end{array}$ & $\begin{array}{l}/ \mathrm{P}_{0} \text { range of } \\
\text { the catalytic }\end{array}$ & \\
\hline
\end{tabular}

activity of the $\mathrm{Pd}(\mathrm{II}) /$ Beta catalyst can be related to the stabilization of smaller nanoparticles with narrower size distribution (see Table 2 and Fig. 3(a), grey diamonds), which can be attributed to the high surface roughness, which is characteristic of this zeolite (see Fig. 4(c)) [49].

For comparison purposes, the same catalysts were prereduced with $\mathrm{NaBH}_{4}$ before performing the catalytic tests, these samples were labelled as $\mathrm{Pd}(0) /$ Zeolite catalysts (Fig. S12). In this case, all the catalysts showed lower activity than their in situ reduced counterparts, see Fig. 3(a) and Fig. S13. However, it is worthy to point out that the same trend was observed between the different zeolites, beta and ferrierite-based catalysts yielding the best results. As in the in situ reduced catalysts, those two zeolites stabilize the smallest Pd NPs and thus maintain narrow particle size distributions (Fig. 3(a), red diamonds), which explains their excellent catalytic performance. Fig. 4 shows the TEM images of the catalysts after reduction with $\mathrm{NaBH}_{4}$ and their corresponding particle size distribution. In most cases, $\mathrm{NaBH}_{4}$ reduction produced higher average nanoparticle size and broader distributions than the catalysts reduced in situ during reaction. This is a trend that has been previously reported for other supports [50]. As described elsewhere, probably an excess of

a)

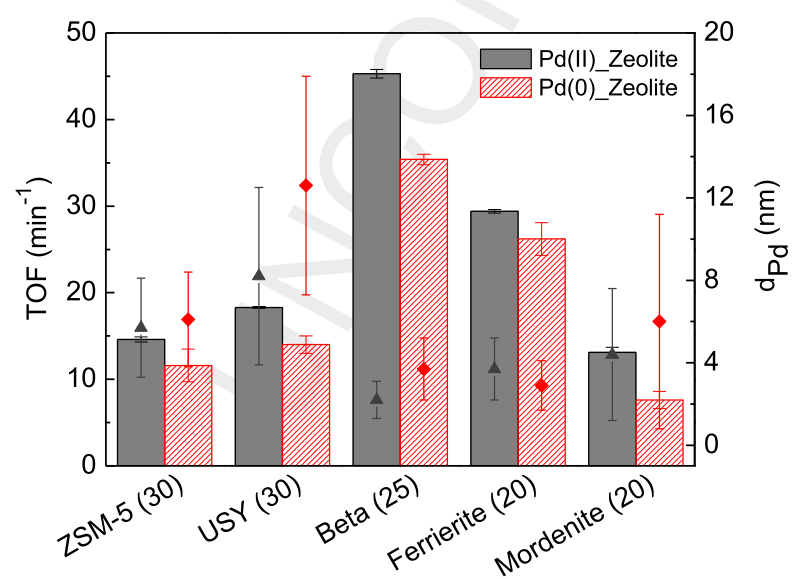

substrate, $A B$ in our case, might be responsible for the stabilization of small Pd NPs under in situ conditions.

Finally, in order to evaluate the influence of the Brønsted acidity of the zeolite in their catalytic performance, the catalyst showing the highest performance (Pd(II)/Beta) was also tested in their ammonium form ( $\mathrm{Pd}(\mathrm{II}) / \mathrm{NH}_{4}$-Beta). It is well known that both Lewis and Brønsted sites coexist in zeolites [51]. The evaluation of the effect of the Brønsted acidity in the reaction requires the removal of the $\mathrm{H}^{+}$by ion-exchange. Fig. 3(b) shows the $\mathrm{H}_{2}$ evolution of catalysts prepared using $\mathrm{H}$-Beta and $\mathrm{NH}_{4}$-Beta and 0.25 wt\% of $\mathrm{Pd}(\mathrm{II})$. As shown, the catalyst with Brønsted acidity offered better catalytic performance, almost doubling the TOF value of the catalyst based on the ammonium form of the zeolite, $45.3 \pm 0.5$ vs $23.8 \pm 1.1$ $\min ^{-1}$, respectively. In the past, acid solids have proven to catalyze this reaction with, however, very low rates when compared to metal catalysts (see Fig. S14 for control experiments with bare zeolites) [23]. Yet, to the best of our knowledge, the bifunctional character of metal/acidic catalysts has not been evaluated to date for the hydrolysis of AB. Nevertheless, previous reports have also stated higher TOF values for Pt and Pd-based catalysts supported on acidic materials, mainly for hydrogenation and hydrogenolysis reactions

b)

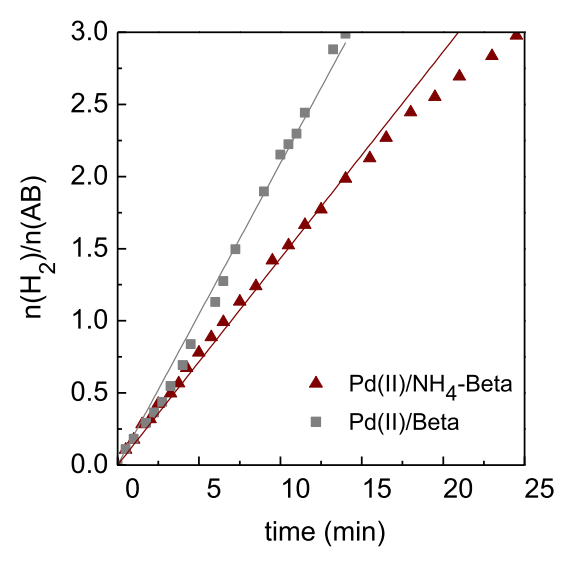



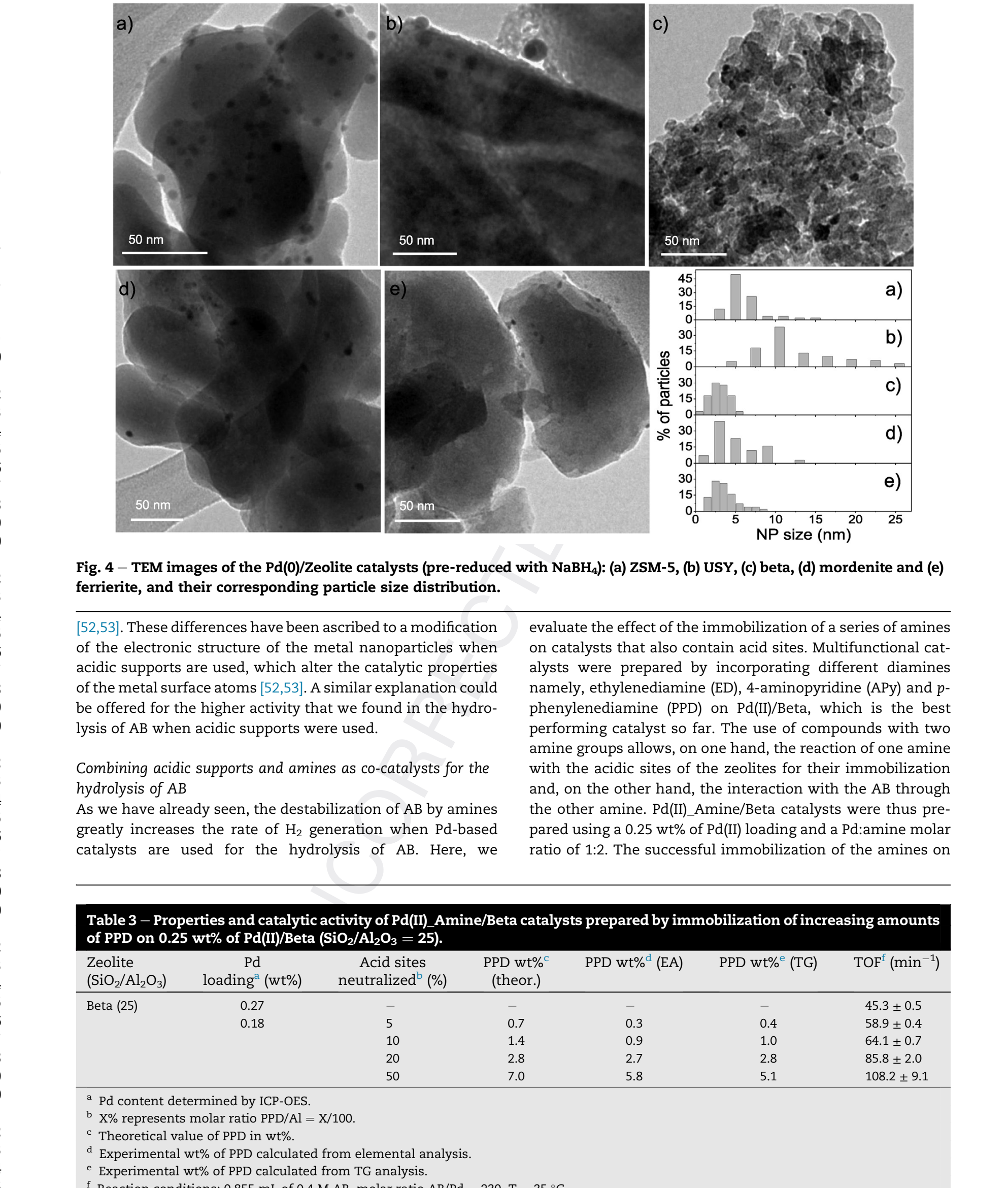

Fig. 4 - TEM images of the Pd(0)/Zeolite catalysts (pre-reduced with $\mathrm{NaBH}_{4}$ ): (a) ZSM-5, (b) USY, (c) beta, (d) mordenite and (e) ferrierite, and their corresponding particle size distribution.
$[52,53]$. These differences have been ascribed to a modification of the electronic structure of the metal nanoparticles when acidic supports are used, which alter the catalytic properties of the metal surface atoms [52,53]. A similar explanation could be offered for the higher activity that we found in the hydrolysis of $A B$ when acidic supports were used.

Combining acidic supports and amines as co-catalysts for the hydrolysis of $A B$

As we have already seen, the destabilization of $A B$ by amines greatly increases the rate of $\mathrm{H}_{2}$ generation when Pd-based catalysts are used for the hydrolysis of $A B$. Here, we evaluate the effect of the immobilization of a series of amines on catalysts that also contain acid sites. Multifunctional catalysts were prepared by incorporating different diamines namely, ethylenediamine (ED), 4-aminopyridine (APy) and $p$ phenylenediamine (PPD) on $\mathrm{Pd}(\mathrm{II}) /$ Beta, which is the best performing catalyst so far. The use of compounds with two amine groups allows, on one hand, the reaction of one amine with the acidic sites of the zeolites for their immobilization and, on the other hand, the interaction with the AB through the other amine. $\mathrm{Pd}(\mathrm{II})$ _Amine/Beta catalysts were thus prepared using a $0.25 \mathrm{wt} \%$ of $\mathrm{Pd}(\mathrm{II})$ loading and a $\mathrm{Pd}$ :amine molar ratio of 1:2. The successful immobilization of the amines on
Table 3 - Properties and catalytic activity of $\mathrm{Pd}($ III)_Amine/Beta catalysts prepared by immobilization of increasing amounts

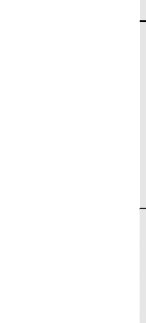


a)

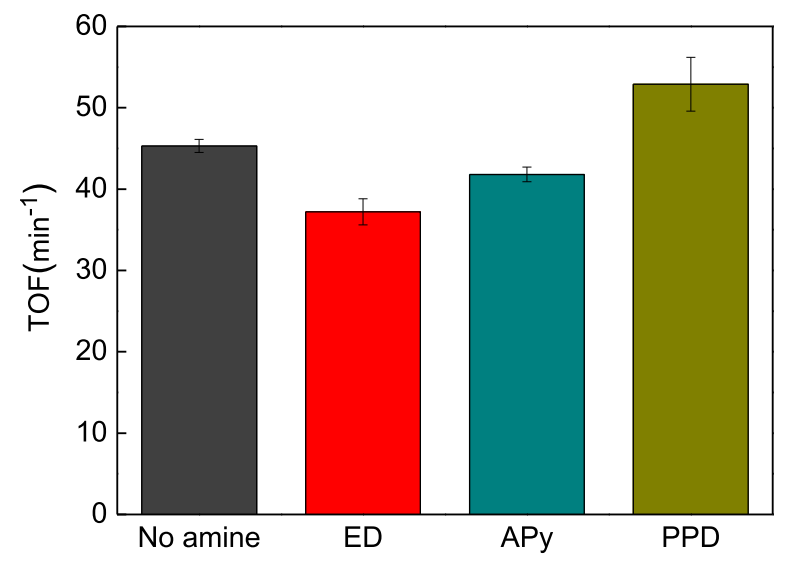

b)

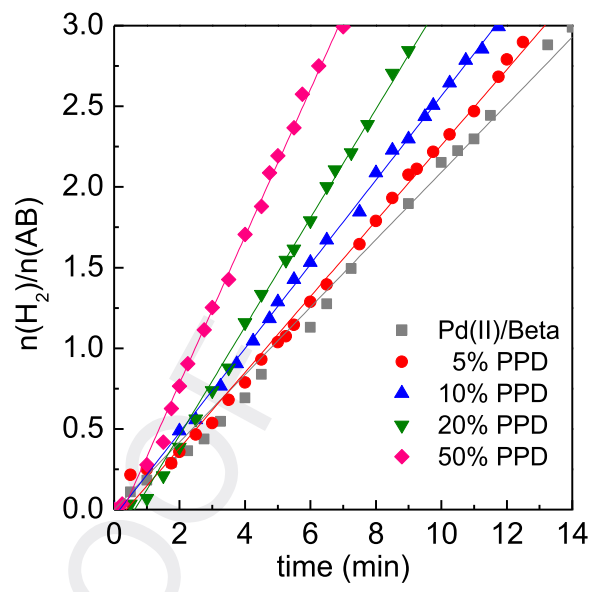

Fig. 5 - (a) TOF values $\left(\mathrm{min}^{-1}\right.$ ) of Pd(II)_Amine/Beta catalysts prepared using a $0.25 \mathrm{wt} \%$ of Pd(II) loading and a Pd:amine molar ratio of 1:2. The different amines used for the functionalization are shown in the $x$ axis. (b) $\mathrm{H}_{2}$ evolution of Pd(II)_PPD/ Beta catalysts functionalized with increasing contents of PPD. Reaction conditions: $0.855 \mathrm{~mL}$ of $0.4 \mathrm{M} \mathrm{AB}, \mathrm{molar}$ ratio AB/ $\mathrm{Pd}=230, \mathrm{~T}=35^{\circ} \mathrm{C}$.

the zeolite was characterized by FTIR, TGA and AE (see Fig. S15 and Table 3). Fig. 5(a) shows the TOF values of the different catalysts evaluated. Similarly to what was observed for the silica support, the only compound that increases the rate of $\mathrm{H}_{2}$ production was the PPD. More specifically, a 20\% enhancement in the rate of $\mathrm{H}_{2}$ generation was obtained when PPD was used in combination with the Pd(II)/Beta catalyst (Fig. S16).

To further explore the effect of combining PPD and Pd(II)/ Beta, a systematic study was carried out in which the PPD content was varied. The acidity of the support plays a key role

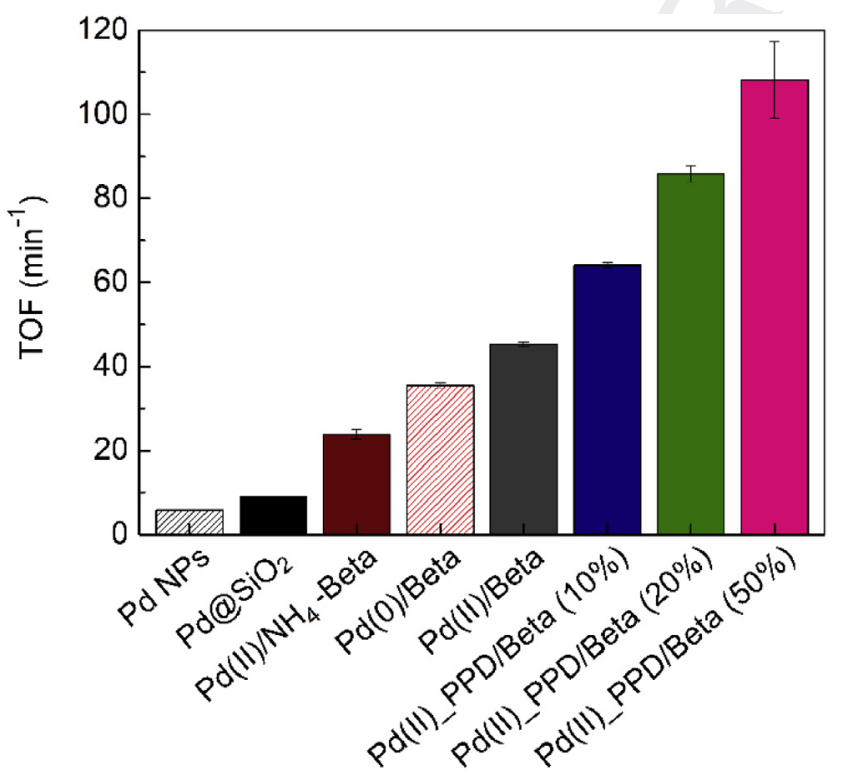

Fig. 6 - Catalytic activity (TOF $\left(\mathrm{min}^{-1}\right)$ ) of a series of Pdbased catalysts prepared introducing different functionalities selected to increase the hydrolysis of AB. All the catalysts were tested under the same reaction conditions. in the reaction and the immobilization of each amine consumes one acid site. Therefore, in order to assess the effect of the PPD incorporation, and taking into account the neutralization of acid sites due to the amine, the PPD was introduced as a percentage, from 5 to $50 \%$, of the acid sites of the zeolite (calculated as the number of $\mathrm{Al}$ present, e.g. 5\% represents: molar ratio $\mathrm{PPD} / \mathrm{Al}=5 / 100)$. As shown in Fig. $5(\mathrm{~b})$, the $\mathrm{H}_{2}$ generation rate directly increases with increasing contents of PPD. It is worth mentioning that the benefit of the incorporation of $\mathrm{PPD}$ is much more pronounced than the negative effect that the neutralization of acid sites could have. Consequently, the incorporation of the highest loading of PPD produces a significant improvement in the catalytic performance, more than doubling the TOF value of the amine-free catalyst, see Table 3.

Finally, Fig. 6 shows a summary of the different TOF values of selected silica- and zeolite-based catalysts prepared in this work. This provides a very clear and visual summary of the positive effect that the addition of each functionality has on the activity of the catalyst. A 10-fold increase in activity has been achieved by: (i) the heterogenization of Pd NPs on an acidic support, (ii) the stabilization of small NPs with narrow particle size distribution by using beta zeolite as support and (iii) the destabilization of $\mathrm{AB}$ by hydrogen bonding with $p$ phenylenediamine.

\section{Recycling of the multifunctional catalysts}

In order to assess the recyclability of our catalysts, five catalytic cycles were performed for the bifunctional $\mathrm{Pd}(\mathrm{II}) /$ Beta and the best performing multifunctional Pd(II)_PPD/Beta (Fig. 7). After each reaction cycle was finished, the catalysts were recovered by centrifugation and reused by addition of new $A B$ aqueous solution. The activity of both catalysts decreased after the first run but they preserve ca. 50\% of the initial TOF value after five catalytic cycles. ICP analyses after 
a)

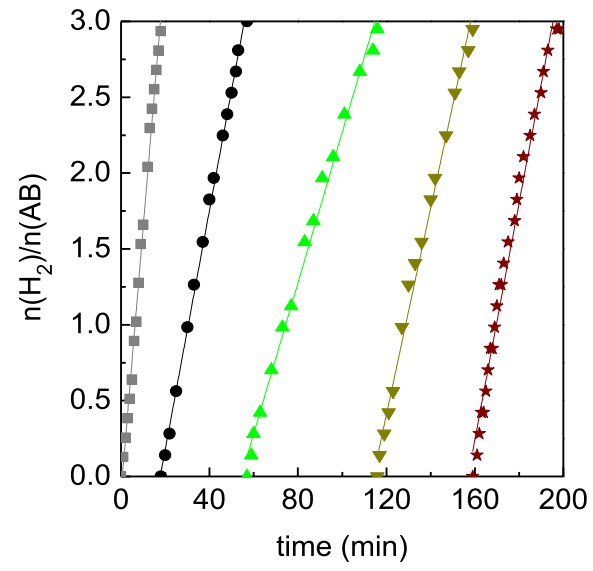

b)

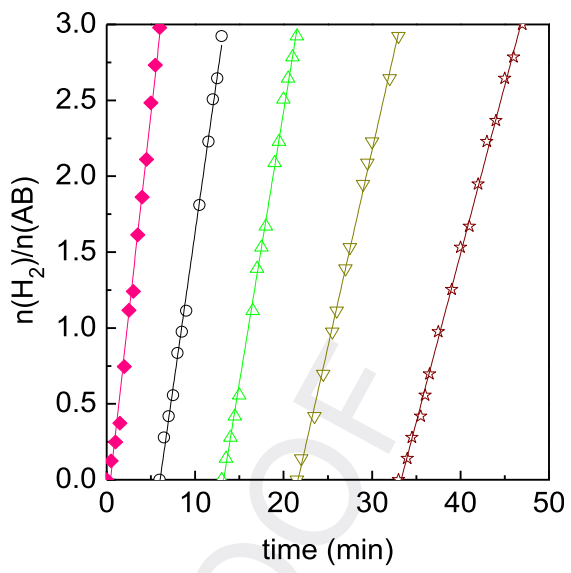

Fig. 7 - Recyclability data. $\mathrm{H}_{2}$ evolution from $\mathrm{AB}$ with (left) the $\mathrm{Pd(II)/Beta} \mathrm{catalyst} \mathrm{and} \mathrm{(right)} \mathrm{the} \mathrm{Pd(II)} \mathrm{PPD/Beta} \mathrm{catalyst.}$ Reaction conditions: $0.4 \mathrm{M} \mathrm{AB}$, molar ratio $\mathrm{AB} / \mathrm{Pd}=230, \mathrm{~T}=35^{\circ} \mathrm{C}$; after each catalytic cycle the catalysts were separated of the reaction mixture by centrifugation before adding new $\mathrm{AB}$ solution.

the first and the last catalytic tests show negligible Pd leaching, which was further confirmed by the lack of catalytic activity of the liquids recovered by centrifugation. The long-term stability of Pd NPs in zeolites has been already reported for this reaction, being a $\mathrm{Pd} / \mathrm{NaY}$ zeolite catalyst able to maintain most of its initial activity after 5 cycles [34]. In our system, the decrease in conversion can be attributed to some aggregation of the Pd NPs but, specially, to the partial loss of the organic compounds. We anticipate that further stabilization of Pd NPs and the organic compounds within the zeolite network, using the different methods developed in our group [54], will further improve the recyclability of the catalyst.

\section{Kinetics of the catalyzed hydrolytic dehydrogenation of $A B$}

In order to determine the activation energy of our system, both the bifunctional Pd(II)/Beta and the multifunctional Pd(II) _PPD/Beta catalysts were studied in the hydrolysis of $A B$ at various temperatures in the $30-55^{\circ} \mathrm{C}$ range, using a concentration of $\mathrm{AB}$ of $0.4 \mathrm{M}$ and a molar ratio $\mathrm{AB} / \mathrm{Pd}=230$. As

a)

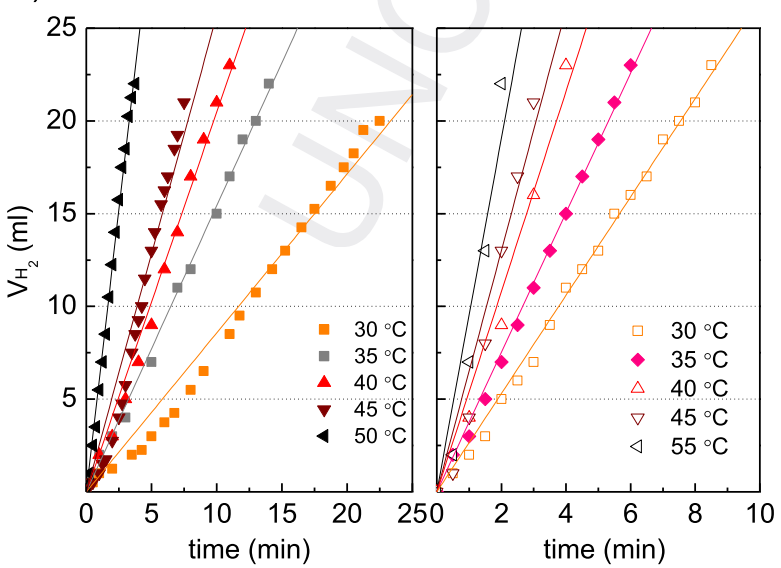

expected, the rate of hydrogen generation increased with the reaction temperature. The values of the rate constant, $\mathrm{k}$, at different temperatures for the catalyzed hydrolysis of $A B$ were calculated from the slope of the linear part of each $\mathrm{H}_{2}$ generation us time plot in Fig. 8(a). These values were used to obtain the Arrhenius plot shown in Fig. 8(b), from which the activation energies, Ea, of both systems were obtained [55]. The presence of PPD causes a significant drop in the apparent Ea for the hydrolysis of $\mathrm{AB}$. The activation energy of the $\mathrm{Pd}(\mathrm{II}) /$ Beta catalyst was determined to be $E a=61 \mathrm{~kJ} \mathrm{~mol}^{-1}$, while the multifunctional system Pd(II)_PPD/Beta presents a lower value, $\mathrm{Ea}=47 \mathrm{~kJ} \mathrm{~mol}^{-1}$, which is one the lowest ever reported for a Pd-based catalyst, see Table $4[56,57]$. The reduction of the activation energy of heterogeneous metal-based catalysts by alloying a particular metal with one or other metals has been widely reported in the literature [58,59]. However, an important contribution of our work is that the kinetic barrier of the $\mathrm{AB}$ hydrolysis was reduced not by introducing a second metal but by using organic compounds. This represents an alternative that is both cost efficient and scalable, and that

b)

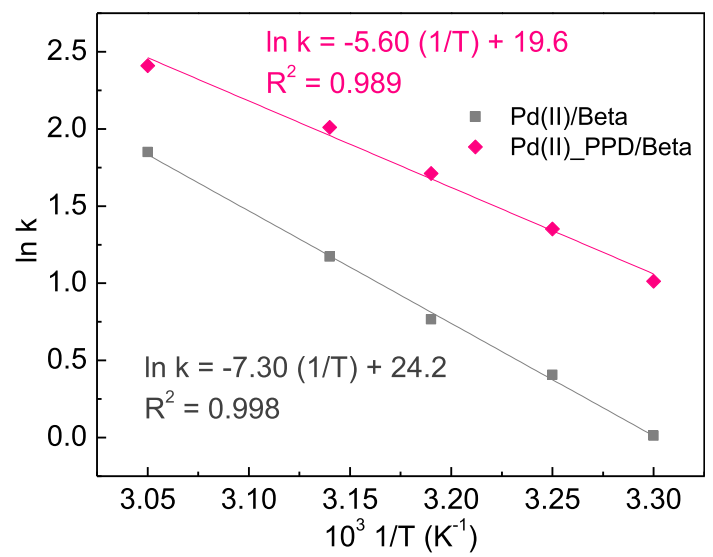

Fig. 8 - (a) Hydrogen generation at different temperatures using (left) the Pd(II)/Beta catalyst and (right) the Pd(II)_PPD/Beta catalyst. (b) The derived Arrhenius plot for both systems. Reaction conditions: $0.4 \mathrm{M} \mathrm{AB}$, molar ratio $\mathrm{AB} / \mathrm{Pd}=230$. 


\begin{tabular}{|c|c|c|c|}
\hline Catalyst & $\begin{array}{c}\mathrm{Ea} \\
\left(\mathrm{kJ} \mathrm{mol}^{-1}\right)\end{array}$ & $\begin{array}{c}\operatorname{TOF}^{[f]} \\
\left(\min ^{-1}\right)\end{array}$ & Reference \\
\hline $\mathrm{Pd} / \mathrm{CoFe}_{2} \mathrm{O}_{4}$ & 42 & 290 & [60] \\
\hline $\mathrm{Pd} / \mathrm{SiO}_{2}-\mathrm{CoFe}_{2} \mathrm{O}_{4}$ & 52 & 254 & [61] \\
\hline $\mathrm{Pd} / \mathrm{PDA}-\mathrm{CoFe}_{2} \mathrm{O}_{4}$ & 65 & 175 & [60] \\
\hline $\mathrm{Pd} / \mathrm{MCN}$ & 57 & 125 & [57] \\
\hline Pd(II)_PPD/Beta & 47 & 55 & This work \\
\hline $\mathrm{Pd} / \mathrm{CeO}_{2}$ & 68 & 29 & [62] \\
\hline $\begin{array}{l}\mathrm{Pd} / \text { chemically } \\
\text { derived graphene }\end{array}$ & - & 15.5 & [63] \\
\hline $\mathrm{Pd} / \mathrm{SiO}_{2}$ & - & 10.0 & [61] \\
\hline 3D Pd/graphene aerogel & 31 & 9.7 & [10] \\
\hline $\mathrm{Pd} / \mathrm{HAP}$ & 55 & 8.3 & [64] \\
\hline $\mathrm{Pd} / \mathrm{NaY}$ & 56 & 6.25 & [34] \\
\hline $\begin{array}{l}\mathrm{Pd} / \text { reduced } \\
\text { graphene oxide }\end{array}$ & 51 & 6.25 & [65] \\
\hline $\begin{array}{l}\text { Colloidal Pd } \\
\text { (PSSA stabilized) }\end{array}$ & 44 & 5 & [66] \\
\hline $\mathrm{Pd} / \mathrm{g}-\mathrm{Al}_{2} \mathrm{O}_{3}$ & - & 1.39 & [67] \\
\hline Pd black & - & 0.67 & [67] \\
\hline
\end{tabular}

can be used in combination with other metals and alloys but also with other supports, which have shown good performances for the hydrolysis of $A B$.

\section{Conclusions}

A series of multifunctional catalysts has been designed, prepared, and tested using different chemical groups that destabilize the $\mathrm{AB}$ molecule as a way to promote its hydrolysis. Our results show that the use of amines in combination with metal sites can significantly improve the performance of the catalysts for the hydrolysis of AB. Specifically, the functionalization of a silica support with $p$-phenylenediamine for the immobilization of Pd NPs produced a three-fold improvement of its catalytic activity. The use of acid sites further boost the hydrolysis of $\mathrm{AB}$. This effect has been analyzed by systematically studying zeolites of different structure and $\mathrm{SiO}_{2} / \mathrm{Al}_{2} \mathrm{O}_{3}$ ratio as supports of Pd NPs. The main conclusions that can be drawn from the characterization and testing of our catalysts are the following:

(i) Catalysts with similar Pd content and nanoparticle size show a slight increase in the hydrogen production rate as the acidity of the zeolite augments. In fact, the TOF values and the relative aluminum content of the zeolite follow a linear trend.

(ii) The presence of Brönsted acid sites in the zeolite support had a pronounced positive effect in their catalytic performance, producing an enhancement of the TOF value of ca. $50 \%$ for the beta-based catalyst, when compared with the zeolite without Brönsted acid sites (NH4-Beta).

(iii) The most important role of the support regarding the hydrolysis of $A B$ is the stabilization of the Pd NPs. Indeed, the best performing catalyst of this work was based on beta zeolite, which high surface roughness helps to maintain the small size of the Pd NPs. (iv) The functionalization of the $\mathrm{Pd}(\mathrm{II}) /$ Beta catalyst with $p$ phenylenediamine yielded the most active catalysts. The kinetics of the catalyzed reaction are directly related to the $p$-phenylenediamine content, as the $\mathrm{H} 2$ generation rate increases linearly with the percentage of $p$-phenylenediamine incorporated. Furthermore, the activation energy of the multifunctional system, $\mathrm{Pd}(\mathrm{II})$ _PPD/Beta, is significantly lower $\left(\mathrm{Ea}=47 \mathrm{~kJ} \mathrm{~mol}^{-1}\right)$ than that of the catalyst without $p$-phenylenediamine, $\mathrm{Pd}(\mathrm{II}) /$ Beta $\left(\mathrm{Ea}=61 \mathrm{~kJ} \mathrm{~mol}^{-1}\right)$,

(v) The use of organic moieties containing amines for the functionalization of the catalysts represents a novel alternative for the reduction of the kinetic barrier on metal-based catalysts for the hydrolysis of $A B$, and therefore to further boost the catalytic performance of other metals and alloys for this reaction.

In summary, by the rational design of a multifunctional catalyst, we have been able to increase the TOF value of the $A B$ hydrolysis of a $\mathrm{Pd@SiO} 2$ catalyst from $8.8 \mathrm{~min}^{-1}$ to $108.2 \mathrm{~min}^{-1}$ for the multifunctional Pd(II)_PPD/Beta system, by using zeolites as supports and readily available organic compounds. This approach is simple and versatile and therefore could be used to improve the performances of other metals and alloys for the hydrolysis of $A B$.

\section{Acknowledgements}

The authors wish to thank the University of Alicante for financial support under the project GRE15-07. E.S. and N.L. acknowledge the University of Alicante, through the "Programa de Retención de Talento" (ref. UATALENTO16-03 and UATALENTO17-05, respectively).

\section{Appendix A. Supplementary data}

Supplementary data related to this article can be found at https://doi.org/10.1016/j.ijhydene.2018.06.137.

\section{REFERENCES}

[1] Demirci UB, Miele P. Sodium borohydride versus ammonia borane, in hydrogen storage and direct fuel cell applications. Energy Environ Sci 2009;2(6):627-37.

[2] Jiang HL, Xu Q. Catalytic hydrolysis of ammonia borane for chemical hydrogen storage. Catal Today 2011;170(1):56-63.

[3] Peng B, Chen J. Ammonia borane as an efficient and lightweight hydrogen storage medium. Energy Environ Sci 2008;1(4):479-83.

[4] Akbayrak S, Özkar S. Ammonia borane as hydrogen storage materials. Int J Hydrogen Energy 2018. https://doi.org/ 10.1016/j.ijhydene.2018.02.190.

[5] Moussa G, Moury R, Demirci UB, Miele P. Borates in hydrolysis of ammonia borane. Int J Hydrogen Energy 2013;38(19):7888-95.

[6] Chandra M, Xu Q. A high-performance hydrogen generation system: transition metal-catalyzed dissociation and 
hydrolysis of ammonia borane. J Power Sources 2006;156(2):190-4.

[7] Xu Q Chandra M. Catalytic activities of non-noble metals for hydrogen generation from aqueous ammonia borane at room temperature. J Power Sources 2006;163(1):364-70.

[8] Kalidindi SB, Indirani M, Jagirdar BR. First row transition metal ion-assisted ammonia borane hydrolysis for hydrogen generation. Inorg Chem 2008;47(16):7424-9.

[9] Akbayrak S, Özkar S. Hydrogen generation from the hydrolysis of ammonia borane using transition metal nanoparticles as catalyst. In: Sankir M, Sankir ND, editors. Hydrogen production technologies, vol. 1. Scrivener Publishing LLC, Wiley; 2017. p. 207-30.

[10] Zhong W d, Tian X k, Yang C, Zhou Z x, Liu X w, Li Y. Active $3 \mathrm{D} \mathrm{Pd} /$ graphene aerogel catalyst for hydrogen generation from the hydrolysis of ammonia-borane. Int J Hydrogen Energy 2016;41(34):15225-35.

[11] Liang H, Chen G, Desinan S, Rosei R, Rosei F, Ma D. In situ facile synthesis of ruthenium nanocluster catalyst supported on carbon black for hydrogen generation from the hydrolysis of ammonia-borane. Int J Hydrogen Energy 2012;37(23):17921-7.

[12] Chandra M, Xu Q. Room temperature hydrogen generation from aqueous ammonia-borane using noble metal nanoclusters as highly active catalysts. J Power Sources 2007;168(1):135-42.

[13] Shen J, Yang L, Hu K, Luo W, Cheng G. Rh nanoparticles supported on graphene as efficient catalyst for hydrolytic dehydrogenation of amine boranes for chemical hydrogen storage. Int J Hydrogen Energy 2015;40(10):1062-70.

[14] Du C, Ao Q, Cao N, Yang L, Luo W, Cheng G. Facile synthesis of monodisperse ruthenium nanoparticles supported on graphene for hydrogen generation from hydrolysis of ammonia borane. Int J Hydrogen Energy 2015;40(18):6180-7.

[15] Wu Y, Wu X, Liu Q, Huang C, Qiu X. Magnetically recyclable $\mathrm{Ni@h-BN} \mathrm{composites} \mathrm{for} \mathrm{efficient} \mathrm{hydrolysis} \mathrm{of} \mathrm{ammonia}$ borane. Int J Hydrogen Energy 2017;42(25):16003-11.

[16] Yamada Y, Yano K, Fukuzumi S. Catalytic application of shape-controlled Cu2O particles protected by $\mathrm{Co} 3 \mathrm{O} 4$ nanoparticles for hydrogen evolution from ammonia borane. Energy Environ Sci 2012;5(1):5356-63.

[17] Yu PJ, Lee MH, Hsu HM, Tsai HM, Chen-Yang YW. Silica aerogel-supported cobalt nanocomposites as efficient catalysts toward hydrogen generation from aqueous ammonia borane. RSC Adv 2015;5(18):13985-92.

[18] Du X, Yang C, Zeng X, Wu T, Zhou Y, Cai P, et al. Amorphous NiP supported on rGO for superior hydrogen generation from hydrolysis of ammonia borane. Int J Hydrogen Energy 2017;42(20):14181-7.

[19] Yan JM, Zhang XB, Akita T, Haruta M, Xu Q. One-step seeding growth of magnetically recyclable Au@Co core-shell nanoparticles: highly efficient catalyst for hydrolytic dehydrogenation of ammonia borane. J Am Chem Soc 2010;132(15):5326-7.

[20] Li Y, Dai Y, Tian X k. Controlled synthesis of monodisperse PdxSn100-x nanoparticles and their catalytic activity for hydrogen generation from the hydrolysis of ammoniaborane. Int J Hydrogen Energy 2015;40(30):9235-43.

[21] Güngörmez K, Metin Ö. Composition-controlled catalysis of reduced graphene oxide supported CuPd alloy nanoparticles in the hydrolytic dehydrogenation of ammonia borane. Appl Catal Gen 2015;494:22-8.

[22] Xia B, Liu C, Wu H, Luo W, Cheng G. Hydrolytic dehydrogenation of ammonia borane catalyzed by metalorganic framework supported bimetallic RhNi nanoparticles. Int J Hydrogen Energy 2015;40(46):16391-7.

[23] Heldebrant DJ, Karkamkar A, Hess NJ, Bowden M, Rassat S, Zheng $\mathrm{F}$, et al. The effects of chemical additives on the induction phase in solid-state thermal decomposition of ammonia borane. Chem Mater 2008;20(16):5332-6.

[24] Stephens FH, Baker R â T, Matus MH, Grant DJ, Dixon DA. Acid initiation of ammonia borane dehydrogenation for hydrogen storage. Angew Chem Int Ed 2007;46(5):746-9.

[25] Zhao J, Shi J, Zhang X, Cheng F, Liang J, Tao Z, et al. A soft hydrogen storage material: poly(methyl acrylate)-confined ammonia borane with controllable dehydrogenation. Adv Mater 2010;22(3):394-7.

[26] Gao L, Li CYV, Yung H, Chan KY. A functionalized MIL-101(Cr) metal-organic framework for enhanced hydrogen release from ammonia borane at low temperature. Chem Commun 2013;49(90):10629-31.

[27] Guo L, Gu X, Kang K, Wu Y, Cheng J, Liu P, et al. Porous nitrogen-doped carbon-immobilized bimetallic nanoparticles as highly efficient catalysts for hydrogen generation from hydrolysis of ammonia borane. J Mater Chem A 2015;3(45):22807-15.

[28] Hu J, Chen Z, Li M, Zhou X, Lu H. Amine-capped Co nanoparticles for highly efficient Dehydrogenation of ammonia borane. ACS Appl Mater Interfaces 2014;6(15):13191-200.

[29] Chandra M, Xu Q. Dissociation and hydrolysis of ammoniaborane with solid acids and carbon dioxide: an efficient hydrogen generation system. J Power Sources 2006;159(2):855-60.

[30] Özgür DÖ, Şimșek T, Özkan G, Akkuș MS, Özkan G. The Hydroloysis of ammonia borane by using Amberlyst-15 supported catalysts for hydrogen generation. Int J Hydrogen Energy 2018.

[31] Rakap M, Özkar S. Hydrogen generation from the hydrolysis of ammonia-borane using intrazeolite cobalt(0) nanoclusters catalyst. Int J Hydrogen Energy 2010;35(8):3341-6.

[32] Zahmakıran M, Ayvalı T, Akbayrak S, Çalışkan S, Çelik D, Özkar S. Zeolite framework stabilized nickel(0) nanoparticles: active and long-lived catalyst for hydrogen generation from the hydrolysis of ammonia-borane and sodium borohydride. Catal Today 2011;170(1):76-84.

[33] Zahmakıran M, Durap F, Özkar S. Zeolite confined copper(0) nanoclusters as cost-effective and reusable catalyst in hydrogen generation from the hydrolysis of ammoniaborane. Int J Hydrogen Energy 2010;35(1):187-97.

[34] Rakap M, Özkar S. Zeolite confined palladium(0) nanoclusters as effective and reusable catalyst for hydrogen generation from the hydrolysis of ammonia-borane. Int $\mathrm{J}$ Hydrogen Energy 2010;35:1305-12.

[35] Zhu QL, Li J, Xu Q. Immobilizing metal nanoparticles to metal organic frameworks with size and location control for optimizing catalytic performance. J Am Chem Soc 2013;135(28):10210-3.

[36] Garcia-Martinez J, Linares N, Sinibaldi S, Coronado E, Ribera A. Incorporation of Pd nanoparticles in mesostructured silica. Microporous Mesoporous Mater 2009;117(1):170-7.

[37] Coronado E, Ribera A, Garcia-Martinez J, Linares N, LizMarzan LM. Synthesis, characterization and magnetism of monodispersed water soluble palladium nanoparticles. J Mater Chem 2008;18(46):5682-8.

[38] Gurav JL, Rao AV, Bangi UKH. Hydrophobic and low density silica aerogels dried at ambient pressure using TEOS precursor. J Alloy Comp 2009;471(1):296-302.

[39] Hilonga A, Kim JK, Sarawade PB, Kim HT. Low-density TEOSbased silica aerogels prepared at ambient pressure using isopropanol as the preparative solvent. J Alloy Comp 2009;487(1):744-50.

[40] Zahmakiran M. Preparation and characterization of LTAtype zeolite framework dispersed ruthenium nanoparticles and their catalytic application in the hydrolytic 
dehydrogenation of ammonia borane for efficient hydrogen generation. Mater Sci Eng, B 2012;177(8):606-13.

[41] Demirci UB, Miele P. Hydrolysis of solid ammonia borane. J Power Sources 2010;195(13):4030-5.

[42] Akdim O, Demirci UB, Miele P. A bottom-up approach to prepare cobalt-based bimetallic supported catalysts for hydrolysis of ammonia borane. Int J Hydrogen Energy 2013;38(14):5627-37.

[43] Brunauer S, Emmett PH, Teller E. Adsorption of gases in multimolecular layers. J Am Chem Soc 1938;60(2):309-19.

[44] Cychosz KA, Guillet-Nicolas R, Garcia-Martinez J, Thommes M. Recent advances in the textural characterization of hierarchically structured nanoporous materials. Chem Soc Rev 2017;46(2):389-414.

[45] Zheng W, Angelopoulos M, Epstein AJ, MacDiarmid AG. Experimental evidence for hydrogen bonding in polyaniline: mechanism of aggregate formation and dependency on oxidation state. Macromolecules 1997;30(10):2953-5.

[46] Stockton WB, Rubner MF. Molecular-level processing of conjugated polymers. 4. Layer-by-Layer manipulation of polyaniline via hydrogen-bonding interactions. Macromolecules 1997;30(9):2717-25.

[47] Auroux A. Acidity characterization by microcalorimetry and relationship with reactivity. Top Catal 1997;4(1):71-89.

[48] Shirazi L, Jamshidi E, Ghasemi MR. The effect of Si/Al ratio of ZSM-5 zeolite on its morphology, acidity and crystal size. Cryst Res Technol 2008;43(12):1300-6.

[49] Navlani-Garcia M, Martis M, Lozano-Castello D, CazorlaAmoros D, Mori K, Yamashita H. Investigation of Pd nanoparticles supported on zeolites for hydrogen production from formic acid dehydrogenation. Catal Sci Technol 2015;5(1):364-71.

[50] Marrodan CM, Berti D, Liguori F, Barbaro P. In situ generation of resin-supported Pd nanoparticles under mild catalytic conditions: a green route to highly efficient, reusable hydrogenation catalysts. Catal. Sci. Technol. 2012;2(11):2279-90.

[51] Derouane EG, Védrine JC, Pinto RR, Borges PM, Costa L, Lemos MAND, et al. The acidity of zeolites: concepts, measurements and relation to catalysis: a review on experimental and theoretical methods for the study of zeolite acidity. Catal Rev 2013;55(4):454-515.

[52] Ramaker DE, de Graaf J, van Veen JAR, Koningsberger DC. Nature of the metal-support interaction in supported Pt catalysts: shift in Pt valence orbital energy and charge rearrangement. J Catal 2001;203(1):7-17.

[53] Miller JT, Mojet BL, Ramaker DE, Koningsberger DC. A new model for the metal-support interaction: evidence for a shift in the energy of the valence orbitals. Catal Today 2000;62(1):101-14.

[54] Serrano E, Linares N, Garcia-Martinez J, Berenguer J. Sol-gel coordination chemistry: building catalysts from the bottomup. ChemCatChem 2013;5(4):844-60.

[55] Özhava D, Kılıçaslan NZ, Özkar S. PVP-stabilized nickel(0) nanoparticles as catalyst in hydrogen generation from the methanolysis of hydrazine borane or ammonia borane. Appl Catal B Environ 2015;162:573-82.

[56] Manna J, Akbayrak S, Ozkar S. Palladium(0) nanoparticles supported on polydopamine coated $\mathrm{Fe} 3 \mathrm{O} 4$ as magnetically isolable, highly active and reusable catalysts for hydrolytic dehydrogenation of ammonia borane. RSC Adv 2016;6(104):102035-42.

[57] Wang W, Lu Z-H, Luo Y, Zou A, Yao Q Chen X. Mesoporous carbon nitride supported Pd and Pd-Ni nanoparticles as highly efficient catalyst for catalytic hydrolysis of NH3BH3. ChemCatChem 2018;10:1620-6.

[58] Du Y, Wang K, Zhai Q, Chen A, Xi Z, Yan J, et al. Alloyed palladium-nickel hollow nanospheres with interatomic charge polarization for improved hydrolytic

dehydrogenation of ammonia borane. Int J Hydrogen Energy 2018;43(1):283-92.

[59] Liu Y, Guan H, Zhang J, Zhao Y, Yang JH, Zhang B. Polydopamine-coated halloysite nanotubes supported AgPd nanoalloy: an efficient catalyst for hydrolysis of ammonia borane. Int J Hydrogen Energy 2018;43(5):2754-62.

[60] Manna J, Akbayrak S, Ozkar S. Palladium(0) nanoparticles supported on polydopamine coated $\mathrm{CoFe}_{2} \mathrm{O}_{4}$ as highly active, magnetically isolable and reusable catalyst for hydrogen generation from the hydrolysis of ammonia borane. Appl Catal B Environ 2017;208:104-15.

[61] Akbayrak S, Kaya M, Volkan M, Ozkar S. Palladium(0) nanoparticles supported on silica-coated cobalt ferrite: a highly active, magnetically isolable and reusable catalyst for hydrolytic dehydrogenation of ammonia borane. Appl Catal B Environ 2014;147:387-93.

[62] Tonbul Y, Akbayrak S, Ozkar S. Palladium(0) nanoparticles supported on ceria: highly active and reusable catalyst in hydrogen generation from the hydrolysis of ammonia borane. Int J Hydrogen Energy 2016;41(26):11154-62.

[63] Metin Ö, Kayhan E, Ozkar S, Schneider JJ. Palladium nanoparticles supported on chemically derived graphene: an efficient and reusable catalyst for the dehydrogenation of ammonia borane. Int J Hydrogen Energy 2012;37(10):8161-9.

[64] Rakap M, Ozkar S. Hydroxyapatite-supported palladium(0) nanoclusters as effective and reusable catalyst for hydrogen generation from the hydrolysis of ammonia-borane. Int J Hydrogen Energy 2011;36(12):7019-27.

[65] Xi P, Chen F, Xie G, Ma C, Liu H, Shao C, et al. Surfactant free RGO/ Pd nanocomposites as highly active heterogeneous catalysts for the hydrolytic dehydrogenation of ammonia borane for chemical hydrogen storage. Nanoscale 2012;4:5597-601.

[66] Metin Ö, Sahin S, Ozkar S. Water-soluble poly(4styrenesulfonic acid-co-maleic acid) stabilized ruthenium(0) and palladium( $(0)$ nanoclusters as highly active catalysts in hydrogen generation from the hydrolysis of ammonia-borane. Int J Hydrogen Energy 2009;34(15):6304-13.

[67] Xu Q Chandra M. A portable hydrogen generation system: catalytic hydrolysis of ammonia-borane. J Alloy Comp 2007;446-447:729-32. 